\title{
Stochastic Charging Coordination Method for Electric Vehicle (EV) Aggregator Considering Uncertainty in EV Departures
}

\author{
Youngwook Kim*, Seongbae Kong* and Sung-Kwan Joo ${ }^{\dagger}$
}

\begin{abstract}
This paper presents a stochastic method for an electric vehicle (EV) aggregator to coordinate EV charging schedule considering uncertainty in EV departures. The EV aggregator is responsible for managing the charging schedule of EVs while participating in the electricity markets. The managed EV charging can provide additional revenues to the aggregator from regulation market participation and charging cost reductions to EV owners. The aggregator needs to coordinate the charging schedule considering various uncertain factors such as electricity market prices and the stochastic characteristics of EVs. In this paper, the EV charging scheduling problem incorporating uncertainty in EV departures is formulated as a stochastic optimization problem. A stochastic optimization method is used to solve the EV charging scheduling problem. Latin hypercube sampling (LHS) and a scenario-reduction method are also applied to reduce the computational efforts of the proposed method. The results of a numerical example are presented to show the effectiveness of the proposed stochastic EV charging coordination method.
\end{abstract}

Keywords: Electrical vehicle, EV aggregator, Stochastic EV departure, Regulation

\section{Introduction}

The recent electricity market allows the participation of the controllable loads as well as conventional generators. Electric vehicles (EVs) have opportunities to participate in the electricity markets through vehicle-to-grid (V2G) or smart charging, i.e., unilateral charging technologies. However, an individual EV has a limited capacity to meet the minimum participating requirements of the electricity markets. The aggregation of electric vehicles (EVs) becomes an attractive alternative solution to encourage the participation of the groups of EVs to the electricity markets. The recent research shows the feasibility of the EV's participation in the markets [1-4].

The aggregate participation of EVs in electricity markets can be accomplished through the qualified EV aggregators by meeting the participating requirements of electricity markets. The EV aggregators can act as an agent for groups of EV owners by controlling the EV charging in order to reduce EV charging cost and receive additional revenue from ancillary services such as frequency regulation. In recent years, there are various attempts [5-7] to determine charging coordination by representing a group of $\mathrm{EV}$ as a fleet with the aggregate characteristics instead of scheduling individual EVs.

When an EV aggregator coordinates EV charging schedules for energy market and ancillary services, the

$\dagger$ Corresponding Author: School of Electrical Engineering, Korea University, South Korea. (skjoo@korea.ac.kr)

* School of Electrical Engineering, Korea University, South Korea ( \{ugi0283, kong8306\}@korea.ac.kr)

Received: February 3, 2015; Accepted: September 2, 2015 aggregator faces the various uncertain factors such as electricity market prices and the stochastic characteristics of EV. In particular, the uncertainty in EV departures can influence the EV charging capability.

The unexpected EV departures during the operation represent a loss of controllable charging capabilities to an aggregator, and the loss of charging capabilities may lead to the shortage of the aggregator's ability to deliver the accepted quantities from the day-ahead electricity markets.

Moreover, the deviation between the day-ahead scheduled EV charging and the actual EV charging could cause extra penalties on EV aggregators depending on market settlement rules [6]. Stochastic approaches are needed to solve the EV coordination problem to handle the uncertainties associated with electricity markets and $\mathrm{EV}$ patterns. However, the most of EV charging coordination methods do not take into account the uncertainties in the EV departures that can lead to the significant loss of the EV controllability to EV aggregators.

This paper presents an EV charging coordination method for an EV aggregator who takes part in day-ahead energy and regulation markets considering the uncertainty in EV departures. The stochastic charging coordination method for an EV aggregator is proposed considering the uncertainty in EV departures. In this study, a group of EVs is represented as an EV fleet with aggregated characteristics, and a scenario-based charging coordination problem is formulated with the modeling of the uncertainties within EV departures. The stochastic optimization technique is used to solve the charging coordination problem. Latin hypercube sampling and fastbackward scenario reduction algorithm are applied as an 
efficient scenario generation method of the unexpected EV departures. The proposed method can help the aggregator to make scheduling decisions regarding the hourly charging rates of EV fleet for the participation in day-ahead energy and regulation markets based on the forecasted electricity market prices.

The remainder of this paper is organized as follows: Section II describes the stochastic EV charging coordination problem. In Section III, the stochastic optimization for the EV charging coordination is formulated, and the scenario generation procedure for stochastic EV departures is presented. Section IV provides the numerical results.

\section{Problem Description}

The increasing number of EVs can provide opportunities for an EV aggregator to participate in electricity markets. The aggregator is an agent for the individual EVs whose battery capacities are too small to participate in electricity markets. Under the agreement between an EV aggregator and EV owners, the owners allow the aggregator to control the charging of EVs for reducing their electricity costs. Fig. 1 shows an overview of the EV aggregator's participation in the energy and regulation markets.

Grouping EVs as a fleet can lower the complexity of the EV coordination problem and provide an effective management method for groups of EVs with the similar characteristics. Based on the historical charging patterns of individual EVs in an EV fleet, the aggregator is able to estimate the characteristics of the fleet, e.g., the arrival and departure times, the number of EV units, and the battery state of charge (SoC) of arriving and departing EVs. These characteristics are used to forecast the EV fleet's behavior and coordinate the charging schedule. Although the EV aggregator could establish the charging schedule under the assumption that EV fleets maintain the constant number of $\mathrm{EV}$ units during the coordination, the unexpected $\mathrm{EV}$

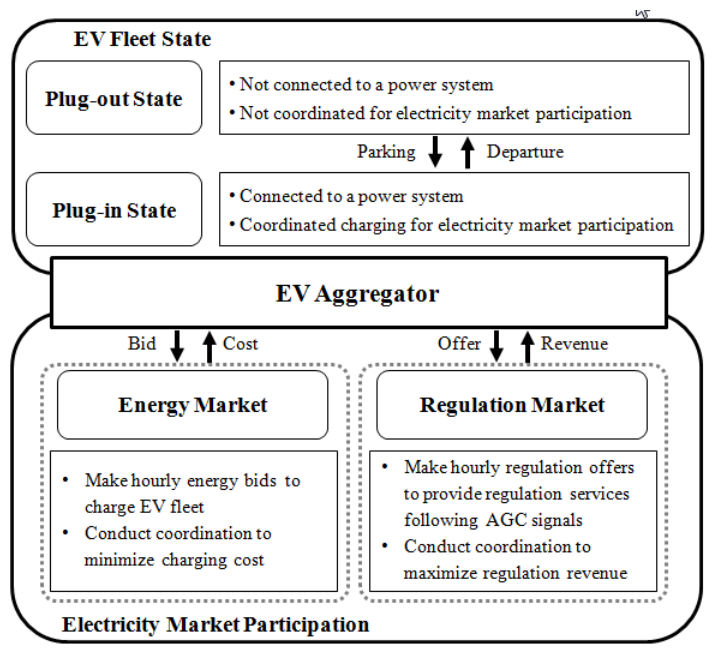

Fig. 1. Electricity market participation of EV aggregator driving behavior can alter the number of charging EVs within a fleet and thus its aggregate charging capabilities.

The unexpected EV departures can result in the significant reduction in the number of controllable EVs within a fleet. The accumulated decrease in the number of controllable EVs during the scheduling time can lead to the substantial loss of the charging capabilities. The shortage of the charging capabilities could prevent EV fleets from maintaining the scheduled charging rates and regulation capacities in actual operation. Moreover, since the deviations from the day-ahead scheduled EV charging may lead to penalties on the aggregator based on market settlement rules, the EV aggregator is required to establish the EV charging coordination for the electricity market participation considering the unexpected EV departures.

\section{Charging Coordination Method Considering Uncertainty in EV Departures}

The EV charging coordination problem for an EV aggregator is formulated as a scenario-based stochastic programming problem in this section. Under the assumption that each EV fleet consists of EVs with similar battery characteristics and driving patterns, the proposed method is designed to determine the hourly energy bids and the regulation offers from the managing EV fleets based on the forecasted day-ahead market prices in order to minimize the overall charging cost. The proposed coordination method takes into account the hourly changes in the number of EVs within a fleet in order to limit the acceptable charging-rate and regulation-capacity ranges considering the uncertainty in EV departures.

The scenario-generation procedure using Latin hypercube sampling (LHS) [8] and a fast-backward scenario reduction algorithm are also described in this section. The uncertainties in the number of departing EVs in a specific fleet are assumed to follow normal distribution. The LHS and scenario reduction techniques are applied to reduce the computational effort of the proposed method.

\subsection{Stochastic EV charging coordination problem}

The objective of the EV charging coordination problem is to determine the charging rates and the regulation capacities in order to minimize the total EV charging cost minus the revenue from regulation as follows:

$$
\operatorname{Min} \sum_{s=1}^{N_{s}} \sum_{t=1}^{N_{T}} \sum_{i=1}^{N_{F}}\left[\rho^{s}\left(M P_{t}^{E} \cdot E_{i, t}^{s}-M P_{t}^{R} \cdot R_{i, t}^{s}\right)\right],
$$

where $M P_{t}^{E}$ and $M P_{t}^{R}$ are the day-ahead forecasted market prices for energy bid and regulation offer at time $t$, respectively; $E_{i, t}^{s}$ and $R_{i, t}^{s}$ are the day-ahead energy bid and regulation offer of fleet $i$ at time $t$ under scenario $s$, 
respectively; $N_{s}, N_{s}$, and $N_{F}$ are the number of scenarios, time periods, and fleets, respectively; $\rho^{s}$ is the occurrence probability of scenario $s$.

The objective function (1) is subject to the following constraints:

\subsubsection{Regulation offer}

The day-ahead regulation offer is equal to the sum of the up and down regulation capacities:

$$
\begin{gathered}
R_{i, t}^{s}=R_{u p, i, t}^{s}+R_{d n, i, t}^{s}, \\
R_{u p, i, t}^{s}=R_{d n, i, t}^{s},
\end{gathered}
$$

where $R_{u p, i, t}^{s}$ and $R_{d n, i, t}^{s}$ are the available up and down regulation capacities of fleet $i$ at time $t$ under scenario $s$, respectively.

The up and down day-ahead regulation of an EV fleet should be provided below its available up and down regulation capacity limits:

$$
\begin{gathered}
R_{u p, i, t}^{s} \leq P_{i, t}^{s}, \\
R_{d n, i, t}^{s} \leq P_{i, t}^{\max , s}-P_{i, t}^{s}
\end{gathered}
$$

where $P_{i, t}^{s}$ represents the average charging power of fleet $i$ at time $t$ under scenario $s$, as follows:

$$
P_{i, t}^{s}=\frac{E_{i, t}^{s}}{\Delta t},
$$

where $\Delta t$ is the duration of time interval $t$. The EV fleet's maximum charging power, $P_{i, t}^{\max , s}$, is assessed from the rated power of the EV charger and the number of pluggedin EVs in the fleet:

$$
P_{i, t}^{\max , s}=P_{i}^{\text {rated }} \cdot N_{i, t}^{E V, s},
$$

where $P_{i}^{\text {rated }}$ is the EV charger's rated power of of fleet $i$. The number of EVs in the fleet, $N_{i, t}^{E V, s}$, is the number of plug-in EVs minus the number of departing EVs from the previous time:

$$
N_{i, t}^{E V, s}=N_{i, t-1}^{E V, s}-N_{d e p, i, t}^{E V, s}
$$

where $N_{d e p, i, t}^{E V, s}$ is the number of the departing EVs of fleet $i$ at time $t$ under scenario $s$.

\subsubsection{Regulation limit}

The energy limits of the up and down regulation during the scheduling horizon are given as follows:

$$
\sum_{\tau=t}^{T_{i}^{d e p}} E_{u p, i, \tau}^{s} \leq \sum_{\tau=t}^{T_{i}^{d e p}}\left(E_{i, \tau}^{s}-E_{u p, i, \tau}^{s}\right),
$$

$$
\begin{aligned}
\sum_{\tau=t}^{T_{i}^{d e p}} E_{d n, i, \tau}^{s} \leq \sum_{\tau=t}^{T_{i}^{d e p}}\left\{E_{i, \tau}^{\max , s}-\left(E_{i, \tau}^{s}+E_{d n, i, \tau}^{s}\right)\right\}, \\
E_{u p, i, \tau}^{s}=R_{u p, i, \tau}^{s} \cdot \Delta t \\
E_{d n, i, \tau}^{s}=R_{d n, i, \tau}^{s} \cdot \Delta t \\
E_{i, \tau}^{\max , s}=P_{i, \tau}^{\max , s} \cdot \Delta t
\end{aligned}
$$

where $E_{u p, i, \tau}^{s}$ and $E_{d n, i, \tau}^{s}$ are the entire energy from the hour-long up and down regulation provisions of fleet $i$ at time $\tau$ under scenario $s$, respectively; $E_{i, \tau}^{\max , s}$ is the maximum charging energy of fleet $i$ at time $\tau$ under scenario $s ; T_{i}^{\text {dep }}$ is the departing time of fleet $i$.

\subsubsection{EV fleet battery SoC}

The state of charge (SoC) of the EV fleet is calculated as follows:

$$
S O C_{i, t}^{s}=S O C_{i, t-1}^{s}+\frac{\eta_{i}}{\operatorname{Cap}_{i, t}^{\text {fleet }, s}} \cdot E_{i, t}^{s}
$$

where $S O C_{i, t}^{s}$ is the SoC of fleet $i$ at time $t$ under scenario $s ; \eta_{i}$ is the charging efficiency of fleet $i$ considering the efficiency between the charger and battery. The overall battery capacity of the EV fleet, Cap fleet,s , is assessed from the battery capacity of the EV unit and the number of EVs in the fleet, as follows:

$$
\operatorname{Cap}_{i, t}^{\text {fleet }, s}=\operatorname{Cap}_{i}^{E V} \cdot N_{i, t}^{E V, s},
$$

where $\operatorname{Cap}_{i}^{E V}$ is the battery capacity of the EV unit in fleet $i$.

The SoC levels of the EV fleet at arrival and departure times are set as follows:

$$
\begin{aligned}
& S O C_{i, T_{i}^{a r r}}=S O C_{i}^{i n i}, \\
& S O C_{i, T_{i}^{d e p}}=S O C_{i}^{f i n},
\end{aligned}
$$

where $S O C_{i, T_{i}^{a r r}}^{s}$ and $S O C_{i, T_{i}^{d e p}}^{s}$ are the SoC levels of fleet $i$ at arrival time and departure time under scenario $s$, respectively; $T_{i}^{\text {arr }}$ is the arrival time of fleet $i ; S O C_{i}^{i n i}$ and $S O C_{i}^{f i n}$ are the initial and final SoCs of fleet $i$, respectively. The SoC of the $\mathrm{EV}$ fleet is maintained between its allowable upper and lower limits:

$$
S O C_{i}^{\min } \leq S O C_{i, t}^{s} \leq S O C_{i}^{\max }
$$

where $S O C_{i}^{\min }$ and $S O C_{i}^{\max }$ are the minimum and maximum SoC of fleet $i$, respectively.

\subsection{EV departure scenario generation}

If the number of the hourly departing EVs is forecasted and the forecasting error is represented as a random 
variable, the number of the unexpectedly departing EVs can be expressed as follows:

$$
N_{d e p, i, t}^{E V}=N_{d e p, i, t}^{E V, f}+\varepsilon_{d e p, i, t}
$$

where $N_{d e p, i, t}^{E V, f}$ is the forecasted departing EV number of fleet $i$ at time $t$, and $\varepsilon_{\text {dep } i, t}$ is a random variable of the forecasting error for the departing EV number of fleet $i$ at time $t$.

If the forecasting error of the departing EV number is normally distributed, the number of unexpectedly departing EVs can be expressed as a normal distribution with a mean and a standard deviation of the forecasted departing EV number:

$$
N_{d e p, i, t}^{E V} \sim N\left(\mu_{d e p, i, t}, \sigma_{d e p, i, t}^{2}\right)
$$

where the parameters of the normal distribution are estimated as follows:

$$
\begin{gathered}
\mu_{d e p, i, t}=N_{d e p, i, t}^{E V, f}, \\
\sigma_{d e p, i, t}^{2}=\sigma_{\varepsilon, i, t}^{2},
\end{gathered}
$$

where $\sigma_{\varepsilon, i, t}^{2}$ is the variance of the departing EV-number forecast error of fleet $i$ at time $t$.

The uncertainty in EV departure is simulated using the optimization model in Section 3.1. The proposed normal distribution in (20) is used to generate the random numbers of the unexpectedly departing EVs over the scheduling horizon, and a large number of scenarios are needed to consider the various probable events. Monte Carlo (MC) simulation using simple random sampling is commonly used for the scenario-based simulation. Due to the large computational effort of the MC simulation, LHS is adopted as a sampling method to generate the stochastic EVdeparture scenarios in this study.

LHS method can effectively cover a probability distribution to generate random numbers. LHS with a Cholesky decomposition can be used to improve the accuracy of the simulation by reducing the possible correlations among the random variable samples [8]. This paper adopts LHS method with Cholesky decomposition as a sampling technique to obtain the random numbers for the generation of EV-departure scenarios. The generated random numbers of departing EVs in the managed EV fleets are modeled to follow the normal distributions that have a cumulative distribution function (CDF) of $F\left(\cdot ; \mu_{d e p, i, t}, \sigma_{d e p, i, t}^{2}\right)$. LHS technique is applied to conduct a random sampling by mapping a sampling map to the inverse CDFs $F^{-1}\left(\cdot ; \boldsymbol{\mu}_{d e p, t}, \boldsymbol{\sigma}_{d e p, t}\right)$ as follows:

$$
\mathbf{N}_{d e p, t}^{E V}=\left[F^{-1}\left(\hat{\mathbf{S}}_{t} ; \boldsymbol{\mu}_{d e p, t}, \boldsymbol{\sigma}_{d e p, t}\right)\right]
$$

where $\hat{\mathbf{S}}_{t}$ is the sampling map; $\boldsymbol{\mu}_{d e p, t}$ and $\boldsymbol{\sigma}_{d e p, t}$ are the mean and the standard deviation parameter vectors of the normal distributions of fleets on the departing EV numbers at time $t$, respectively; $[\cdot]$ is the round function that returns the nearest integer.

Since the random samples are generated hourly for the managed EV fleets during the scheduling time periods, the charging coordination problem becomes a multidimensional and multistage problem. A large number of scenarios are simulated and, in turn, require substantial computation efforts to simulate all the generated scenarios. Fast-backward scenario reduction algorithm is an effective method to reduce the number of scenarios by selecting a subset from the original scenario set through the recursive deleting procedure [9]. In this paper, the fast-backward scenario reduction algorithm is adopted as the scenarioreduction technique to solve the large-scale scenario-based optimization problem efficiently. The target scenario set to which the scenario-reduction technique is applied is the collection of original EV-departure scenarios generated by LHS method. The application of the scenario-reduction technique can help reduce the substantial computational efforts involved in handling a large number of $\mathrm{EV}$ departure scenarios generated by LHS during the simulation.

\section{Numerical Results}

This section presents the numerical results to demonstrate the effectiveness of the proposed EV charging coordination method. The proposed coordination method is compared with the deterministic coordination approach in Section 3.1.

The deterministic approach attempts to determine the hourly charging rates and regulation capacities with assumption that a constant number of EVs is maintained during the scheduling horizon.

The numerical tests for the deterministic and proposed coordination method are conducted on an EV aggregator that manages two EV fleets, i.e. EV fleet 1 and EV fleet 2. The managed EVs within those fleets are assumed to be used for commuting purposes. The EV owners may park their EVs during the work period, and the aggregator coordinates the charging rates of the parked EVs to participate in the day-ahead electricity markets. According to the U.S. Department of Transportation's National Household Travel Survey, the trips of the vehicles for commutes to work occur mainly between 6:00 and 9:00 in the morning, and those vehicles are often parked for seven or more hours at the workplace [10]. It is assumed in this study that charging coordination would be conducted for the EVs that are plugged in between 9:00 a.m. and 5:00 p.m. during a day following the survey result.

It is also assumed that each EV fleet consists of a group of EVs with identical battery characteristics. EV fleet 1 and 
2 are assumed to include 1,000 units with $24-\mathrm{kWh}$ Li-ion battery and 500 units with $85-\mathrm{kWh}$ Li-ion battery, respectively. The batteries operate in the SoC range from $20 \%$ to $80 \%$. The initial SoC levels of a $24-\mathrm{kWh}$ and $85-$ $\mathrm{kWh}-\mathrm{EV}$-fleet are assigned as $40 \%$ and $60 \%$, respectively. The target SoCs of the both fleets are set as $80 \%$. The SoCs of the EV fleets are assumed to increase in proportion to the hourly charging power. The distinct characteristics of EV fleet 1 and EV fleet 2 are summarized in Table 1.

EV fleets 1 and 2 for the numerical test are charged through Level-2 chargers which are used for the nonresidential EV charging. The Level-2 charger can provide charging power from $3.3 \mathrm{~kW}$ to $7.7 \mathrm{~kW}$. It is assumed that the EVs of the fleet 1 are charged only through a Level-2 charger with 3.3-kWh rated power, and that the EVs of the fleet 2 are charged with $6.6-\mathrm{kWh}$ rated power. The charging efficiency is set as $83.7 \%$ following the result of an EV-charging field test [11].

In addition to modeling the EV fleets, the scenarios of the forecasted day-ahead market prices and the hourly EV departures within the fleets are required for the numerical test. This paper uses the average historical data of NYISO's day-ahead energy and regulation market prices as the scenario of the forecasted day-ahead market prices. Fig. 2 shows the average energy and regulation prices of NYISO's electricity markets for one month (January 2014). LHS technique and fast-backward scenario reduction algorithm in Section 3.2 are applied to generate the scenarios of the unexpectedly departing EV numbers.

LHS generates the EV departure scenarios using the hourly normal distributions of the forecasted departing EV numbers. Since the rate of vehicle trips for a commute from office to home substantially increases as the time gets closer to $17: 00$ [10], it is assumed in this study that the mean and the standard deviation of the normal distributions

Table 1. Characteristics of EV fleets

\begin{tabular}{c|c|c|c}
\hline No. & $\begin{array}{c}\text { Number of } \\
\text { EVs }\end{array}$ & $\begin{array}{c}\text { Battery capacity } \\
(\mathrm{kWh})\end{array}$ & $\begin{array}{c}\text { Initial SoC } \\
(\%)\end{array}$ \\
\hline EV fleet 1 & 1,000 & 24 & 40 \\
\hline EV fleet 2 & 500 & 85 & 60 \\
\hline
\end{tabular}

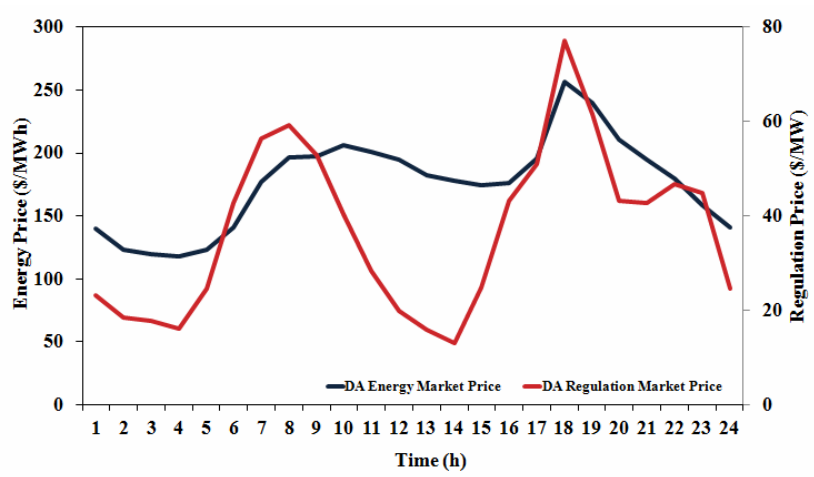

Fig. 2. Day-ahead hourly mean values of regulation and energy prices increase after 15:00. After the EV departure scenarios are generated, the fast-backward scenario reduction algorithm is applied to obtain the subsets from the generated scenarios. Fig. 3 shows the 50 generated scenarios for the hourly departing EV numbers from 10:00 to 17:00 using LHS and the scenario reduction algorithm.

Table 2 shows the results of the overall coordination cost for the numerical tests of the proposed and deterministic methods. Compared with the deterministic method, the unexpected EV departures of the proposed method affect the number of energy bids and regulation offers in a decreasing direction.

When the proposed method is applied, the total coordination cost decreases from $\$ 3,136$ to $\$ 2,665$ because of the larger reduction in charging cost than regulation revenue. The decreases in charging cost and regulation revenue between the proposed method and the deterministic method are $16.15 \%$ and $20.26 \%$, respectively.

The hourly energy bids and regulation offers for the numerical tests are shown in Fig. 4 and Fig. 5 When the deterministic method is applied, the EVs are charged from 10:00 to 11:00 and again from 14:00 to 17:00 in Fig. 4. The amount of energy bids is maximized at 16:00 since the lowest energy price is forecasted and the certain amount of profit from the regulation provision is obtained. The overall regulation offers occur at the same time as the energy bids, except at 14:00 when the cost for EV charging is relatively high and the market price for regulation offers is low. The regulation offers are concentrated at 10:00 and 17:00 when the regulation market prices are high in Fig. 5.

The numerical results from the proposed method in Fig. 4 and Fig. 5 show a decrease in both energy bids and regulation offers compared to those of the deterministic method. The continuous EV departures during the

Table 2. Comparison of proposed and deterministic methods in coordination cost

\begin{tabular}{c|c|c}
\hline Coordination cost & $\begin{array}{c}\text { Deterministic } \\
\text { method }(\$)\end{array}$ & $\begin{array}{c}\text { Proposed } \\
\text { method }(\$)\end{array}$ \\
\hline $\begin{array}{c}\text { Charging cost } \\
\text { Regulation revenue }\end{array}$ & $\begin{array}{c}3,995 \\
859(\mathrm{credit})\end{array}$ & $\begin{array}{c}3,350 \\
685(\mathrm{credit})\end{array}$ \\
\hline Total cost & 3,136 & 2,665 \\
\hline
\end{tabular}

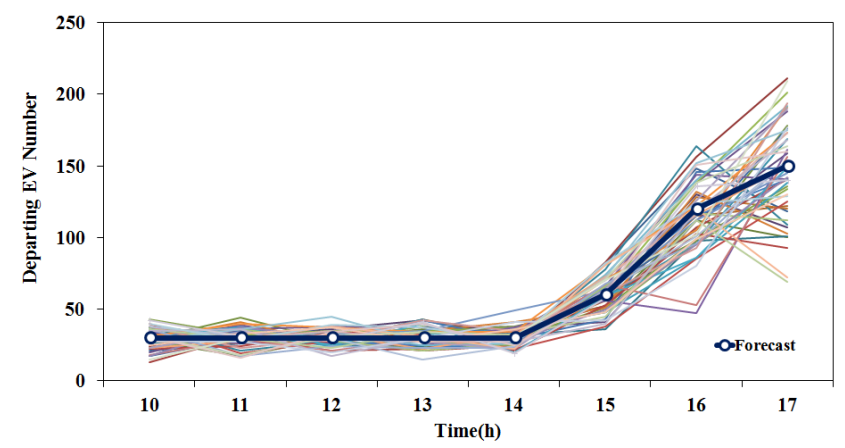

Fig. 3. Scenario-generation results of hourly EV departures 
scheduling time periods affect the amount of hourly energy bids and regulation offers at 11:00 and again from 13:00 to 17:00. Noticeable decreases in energy bids mainly occur after 15:00 in Fig. 4 because of a reduction in charging demand that is caused by an increasing number of EV departures. A reduction in regulation offers also occurs at 11:00, 15:00, and 17:00 according to Fig. 5. The largest reduction at 17:00 is mainly due to the decrease in the number of plug-in EVs and the loss of their EV chargers that can provide controllable charging capabilities for regulation. The parts of the reduced regulation are shifted at 13:00, 14:00, and 16:00 where extra capacities for regulation can be obtainable. Fig. 6 shows the hourly

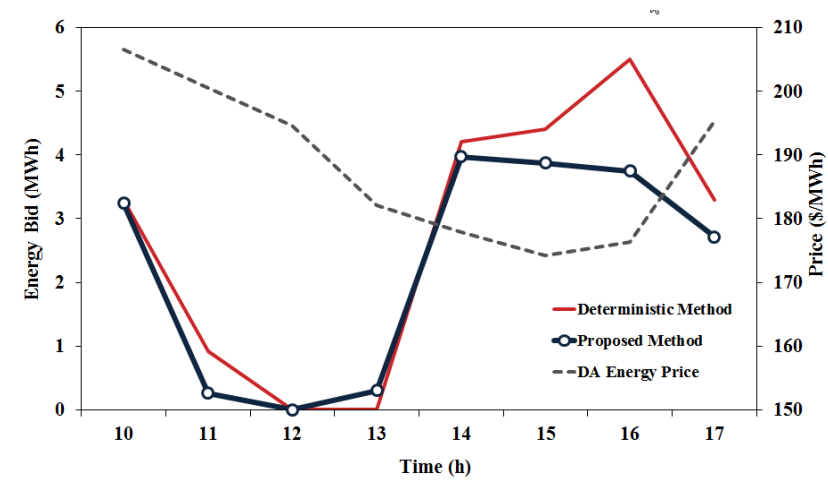

Fig. 4. Coordinated hourly energy bids of proposed and deterministic methods

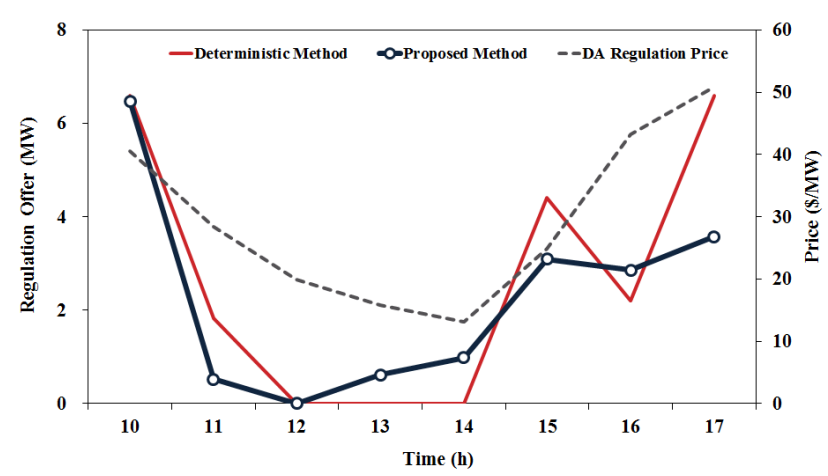

Fig. 5. Coordinated hourly regulation offers of proposed and deterministic methods

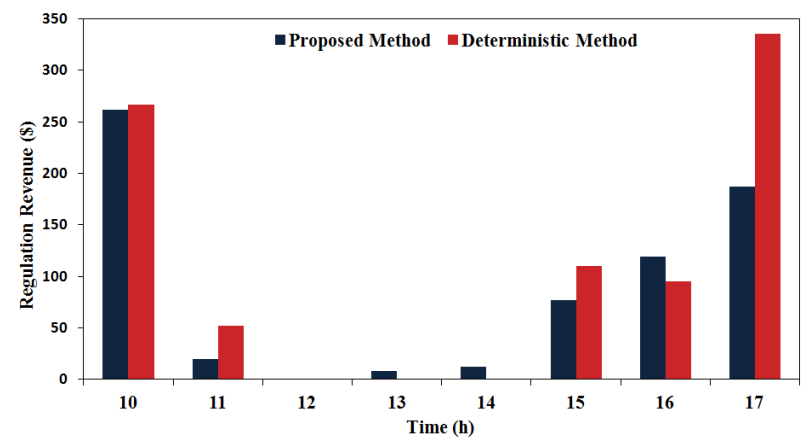

Fig. 6. Hourly regulation revenues of proposed and deterministic methods regulation revenues from both the proposed and deterministic methods.

The numerical test results of the proposed and deterministic methods show that, if an EV aggregator performs the EV charging coordination without considering the accumulated EV departures during the scheduling time periods, the EV aggregator determines the hourly dayahead energy bids and regulation offers of which the aggregator cannot deliver the scheduled quantities during the real-time operation due to the shortage of the controllable charging capabilities of EVs.

In this study, the proposed EV charging scheduling method is designed to charge EVs at the target SoC levels given the expected EV arrival and departure times. The proposed method determines the hourly charging rates and regulation capacities of $\mathrm{EV}$ fleets in order to minimize the overall coordination cost according to the scenarios of the forecasted electricity market prices and the unexpected EV departures. The coordination results show that the EV fleets would be charged at their target SoC levels between the arrival and departure times.

In practice, the EV departures could unexpectedly occur in the middle of charging. In spite of the earlier departure than the scheduled time the EV owners would still anticipate that their EV is charged at a certain SoC level during the parking periods. The proposed method can provide an EV aggregator with the cost-effective coordination solution from the point of view of least coordination cost, but it may not ensure that the SoC of EVs increase in proportion to parking periods.

Charging EVs at constant rates can increase SoC levels in proportional to their plug-in times and thus become an effective charging strategy from the point of view of the $\mathrm{EV}$ owner's convenience. A numerical test is conducted for the identical scenario set with that of the proposed method in order to show the effects of the constant EV charging on the charging coordination problem.

It is assumed under the constant charging method that the EV fleet 1 and 2 are charged with constant rates 1.3 $\mathrm{MWh}$ and 1.4 MWh from 10:00 to 13:00, respectively. Then, the hourly regulation capacities for 8 hours and charging rates from $14: 00$ to $17: 00$ are determined following the optimization model described in Section 3.1.

Table 3 shows the coordination cost when the constant charging method is applied. Compared with the coordination cost result of the proposed method in Table 2, the total cost is increased from $\$ 2,665$ to $\$ 2,880$ due to the larger increase in the charging cost than in the regulation revenue. The constant charging between 11:00 and 13:00 causes the significant increase in the charging cost while the proposed

Table 3. Coordination cost of constant charging method

\begin{tabular}{c|c|c|c}
\hline $\begin{array}{c}\text { Coordination } \\
\text { cost }\end{array}$ & $\begin{array}{c}\text { Charging } \\
\text { cost }\end{array}$ & $\begin{array}{c}\text { Regulation } \\
\text { revenue }\end{array}$ & Total cost \\
\hline $\begin{array}{c}\text { Constant charging } \\
\text { method }(\$)\end{array}$ & 3,537 & 717 (credit) & 2,820 \\
\hline
\end{tabular}




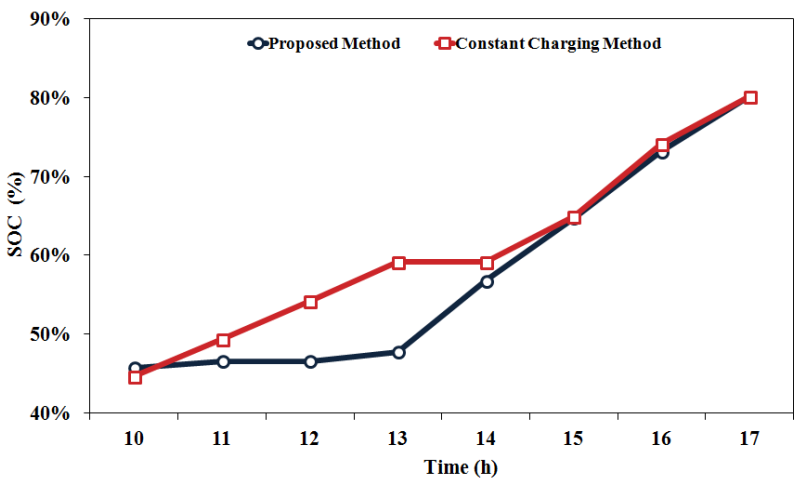

Fig. 7. Hourly SoC levels of EV fleet 1 obtained by proposed and constant charging methods

method schedules the EV charging apart from those time periods of high charging cost and relatively low regulation revenue.

Fig. 7 shows the hourly SoC results of EV fleet 1 for the proposed and constant charging methods. While the proposed method increases the SoC of EV fleet 1 less than $10 \%$ until 13:00, the constant charging method continuously increase the SoC of fleet 1 except 14:00 and the SoC level reaches close to $60 \%$ before 14:00. The SoC result shows that the constant charging method can provide the EV owners with the more satisfaction from the point of view of the EV owner's convenience than the proposed method for the SoC levels of the departing EVs that leave in the middle of the EV charging schedule.

It can be seen from the numerical results that the uncertainty in $\mathrm{EV}$ departures has impacts on the EV charging coordination due to the decrease in the hourly number of the plug-in EVs during the scheduling time periods and the proposed stochastic coordination method forces the amount of hourly charging rates and regulation capacities to be shifted or reduced considering the decreasing number of plug-in EVs, otherwise cause the shortage of the controllable charging capabilities to provide the scheduled energy charging rates and regulation capacities.

Furthermore, the proposed method can be used in combination with the constant charging strategy for some EV owners who select higher EV charging tariff rate plan at time of parking to avoid their inconvenience. The constant charging strategy can be applied up to the preferred minimum SoC charging level established by those EV owners.

\section{Conclusion}

An EV aggregator can participate in the day-ahead electricity markets using aggregate EVs for energy bids and the provision of ancillary services. This paper presents a stochastic EV charging coordination method for the EV aggregator to coordinate the charging of EV fleets considering uncertainty in EV departures. The proposed method is developed under the assumption that an EV aggregator participates in both the energy and regulation electric markets. The LHS technique and a fast-backward scenario reduction algorithm are applied to reduce the computational effort of the proposed method.

\section{Acknowledgements}

This research was supported by Basic Science Research Program through the National Research Foundation of Korea (NRF) funded by the Ministry of Education, Science and Technology (2013R1A1A2057969). This work was supported by "Human Resources program in Energy Technology" of the Korea Institute of Energy Technology Evaluation and Planning(KETEP) granted financial resource from the Ministry of Trade, Industry \& Energy, Republic of Korea (No. 20154030200610).

\section{References}

[1] B. Jansen, C. Binding, O. Sundström, and D. Gantenbein, "Architecture and Communication of an Electric Vehicle Virtual Power Plant," in IEEE Proc. First IEEE International Conference on Smart Grid Communications, 2010, pp. 149-154.

[2] C. Binding, D. Gantenbein, B. Jansen, O. Sundström, P. B. Andersen, F. Marra, B. Poulsen, and C. Traeholt, "Electric Vehicle Fleet Integration in the Danish Edison Project - A Virtual Power Plant on the Island of Bornholm," IEEE Power and Energy Society General Meeting, 2010, pp. 1-8.

[3] W. Kempton, V. Udo, K. Huber, K. Komra, S. Letendre, S. Baker, D. Brunner, and N. Pearre, "A Test of Vehicle-to-grid (V2G) for Energy Storage and Frequency Regulation in the PJM System," University of Delaware, Tech. Rep., Nov. 2008.

[4] J. U. Lee, Y. M. Wi, Y. Kim, and S. K. Joo, “Optimal Coordination of Charging and Frequency Regulation for an Electric Vehicle Aggregator Using Least Square Monte-Carlo (LSMC) with Modeling of Electricity Price Uncertainty," Journal of Electrical Engineering and Technology, Vol. 8, No. 6, pp. 12691275, Nov. 2013.

[5] S. I. Vagropoulos and A. G. Bakirtzis, "Optimal Bidding Strategy for Electric Vehicle Aggregators in Electricity Markets," IEEE Trans. Power Systems, vol. 28, no. 4, pp. 4031-4041, Nov. 2013.

[6] R. J. Bessa and M. A. Matos, "Optimization Models for an EV Aggregator Selling Secondary Reserve in the Electricity Market," Electric Power Systems Research, vol. 106, pp. 36-50, Jan. 2014. 
[7] R. J. Bessa, M. A. Matos, F. J. Soares, and J. A. P. Lopes, "Optimized Bidding of a EV Aggregation Agent in the Electricity Market," IEEE Trans. Smart Grid, vol. 3, no. 1, pp. 443-452, Mar. 2012.

[8] A. Olsson, G. Sandberg, and O. Dahlblom, "On Latin Hypercube Sampling for Structural Reliability Analysis," Structural Safety, vol. 25, no. 11, pp. 4768, Jan. 2003.

[9] H. Heitsch and W. Romisch, "Scenario Tree Reduction for Multistage Stochastic Programs," Compu. Manag. Scien., vol. 6, no. 2, pp. 117-133, May 2009.

[10] A. Santos, N. McGuckin, H. Nakamoto, D. Gray, and S. Liss, "Summary of Travel Trends: 2009 National Household Travel Survey," U.S. Department of Transportation, Federal Highway Administration, Tech. Rep., Jun. 2011.

[11] E. Forward, K. Glitman, and D. Roberts, "An Assessment of Level 1 and Level 2 Electric Vehicle Charging Efficiency," EVT NRA R\&D Electric Vehicle Supply Equipment Project Report, Jun. 2013.

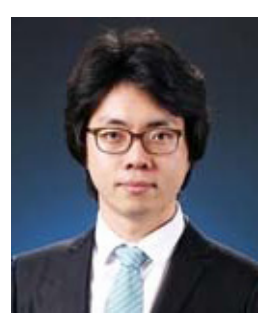

Youngwook Kim He received B.S. degree from Korea University, Seoul, Republic of Korea, in 2010. He is currently pursuing Ph.D. degree in Electrical Engineering at Korea University.

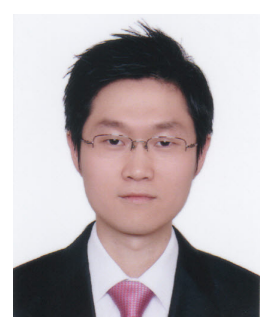

Seongbae Kong He received M.S. degree from Korea University, Seoul, Republic of Korea, in 2011. He is currently pursuing Ph.D. degree in Electrical Engineering at Korea University.

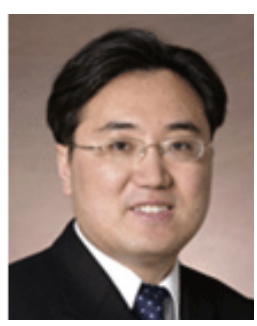

Sung-Kwan Joo He received the M.S. and Ph.D. degrees from the University of Washington, Seattle, in 1997 and 2004, respectively. He is currently a Professor in the school of electrical engineering at Korea University, Seoul, Korea. 